\title{
Stacking in Colloidal Nanoplatelets: Tuning Excitonic Properties
}

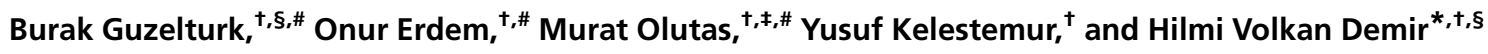 \\ ${ }^{\dagger}$ Department of Electrical and Electronics Engineering, Department of Physics, UNAM - Institute of Materials Science and Nanotechnology, Bilkent University, \\ Ankara 06800, Turkey, ${ }^{\ddagger}$ Department of Physics, Abant Izzet Baysal University, Bolu 14280, Turkey, and ${ }^{\S}$ Luminous! Center of Excellence for Semiconductor Lighting \\ and Displays, School of Electrical and Electronic Engineering, School of Physical and Mathematical Sciences, Nanyang Technological University, Nanyang Avenue, \\ Singapore 639798, Singapore. ${ }^{\# B}$. Guzelturk, 0. Erdem, and M. Olutas contributed equally to this work.
}

\begin{abstract}
Colloidal semiconductor quantum wells, also commonly known as nanoplatelets (NPLs), have arisen among the most promising materials for light generation and harvesting applications. Recently, NPLs have been found to assemble in stacks. However, their emerging characteristics essential to these applications have not been previously controlled or understood. In this report, we systematically investigate and present excitonic properties of controlled column-like NPL assemblies. Here,
\end{abstract}
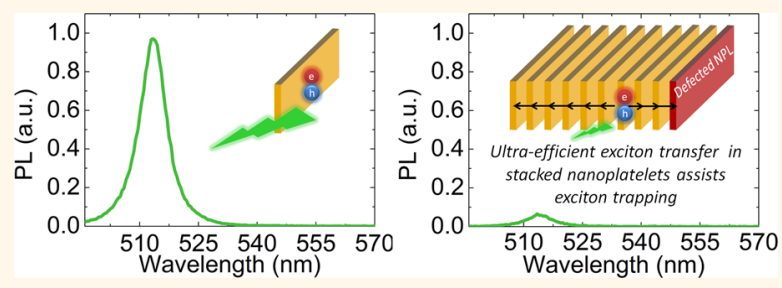

by a controlled gradual process, we show that stacking in colloidal quantum wells substantially increases exciton transfer and trapping. As NPLs form into stacks, surprisingly we find an order of magnitude decrease in their photoluminescence quantum yield, while the transient fluorescence decay is considerably accelerated. These observations are corroborated by ultraefficient Förster resonance energy transfer (FRET) in the stacked NPLs, in which exciton migration is estimated to be in the ultralong range ( $>100 \mathrm{~nm}$ ). Homo-FRET (i.e., FRET among the same emitters) is found to be ultraefficient, reaching levels as high as $\mathbf{9 9 . 9 \%}$ at room temperature owing to the close-packed collinear orientation of the NPLs along with their large extinction coefficient and small Stokes shift, resulting in a large Förster radius of $\sim 13.5 \mathrm{~nm}$. Consequently, the strong and long-range homo-FRET boosts exciton trapping in nonemissive NPLs, acting as exciton sink centers, quenching photoluminescence from the stacked NPLs due to rapid nonradiative recombination of the trapped excitons. The rate-equation-based model, which considers the exciton transfer and the radiative and nonradiative recombination within the stacks, shows an excellent match with the experimental data. These results show the critical significance of stacking control in NPL solids, which exhibit completely different signatures of homo-FRET as compared to that in colloidal nanocrystals due to the absence of inhomogeneous broadening.

KEYWORDS: colloidal quantum wells · colloidal nanoplatelets · nonradiative energy transfer · Förster resonance energy transfer · time-resolved fluorescence spectroscopy $\cdot$ exciton trapping

In $\mathrm{n}$ the past two decades colloidal semiconductor nanostructures have emerged as thriving materials thanks to the ease of their colloidal synthesis along with their unique optical and electronic properties, making these materials competitively advantageous in various fields ranging from optoelectronics to bioimaging and sensing. ${ }^{1-3}$ Now colloidal semiconductor nanostructures having various shape, size, and composition can be epitaxially synthesized with very high quality. ${ }^{4-7}$ Recently, colloidal semiconductor quantum wells, also more commonly known as nanoplatelets (NPLs), have been introduced. NPLs possess strong quantum confinement only in one dimension (vertical direction), resembling epitaxial quantum wells. ${ }^{8}$ The NPLs exhibit favorable optical properties including a narrow emission full-width at half-maximum (fwhm) of $\sim 30 \mathrm{meV}$ at room temperature owing to the absence of inhomogeneous broadening, a shorter radiative lifetime, and a giant oscillatory strength. ${ }^{9-15}$ These properties have already made NPLs highly attractive for light-generation applications including lasers and light-emitting diodes (LEDs). ${ }^{16-18}$

Controlling the nanoscale assembly of the NPLs and understanding their emerging properties are crucial in light-generationbased applications. Previously, stacking of the colloidal NPLs has been reported both in solution and in solid films. ${ }^{19,20}$ Stacked NPL assemblies exhibit different optical properties as compared to the nonstacked ones. For instance, the low-temperature photoluminescence spectrum of the stacked NPLs was shown to differ from that of the nonstacked NPLs by the phonon-line emission
*E-mail:

volkan@bilkent.edu.tr,

hvdemir@ntu.edu.sg. Phone: +90 312 290-

1021. Fax: +90312 290-1123.

Received for review September 22, 2014 and accepted December 3, 2014.

Published online December 03, 2014 $10.1021 /$ nn5053734

() 2014 American Chemical Society 

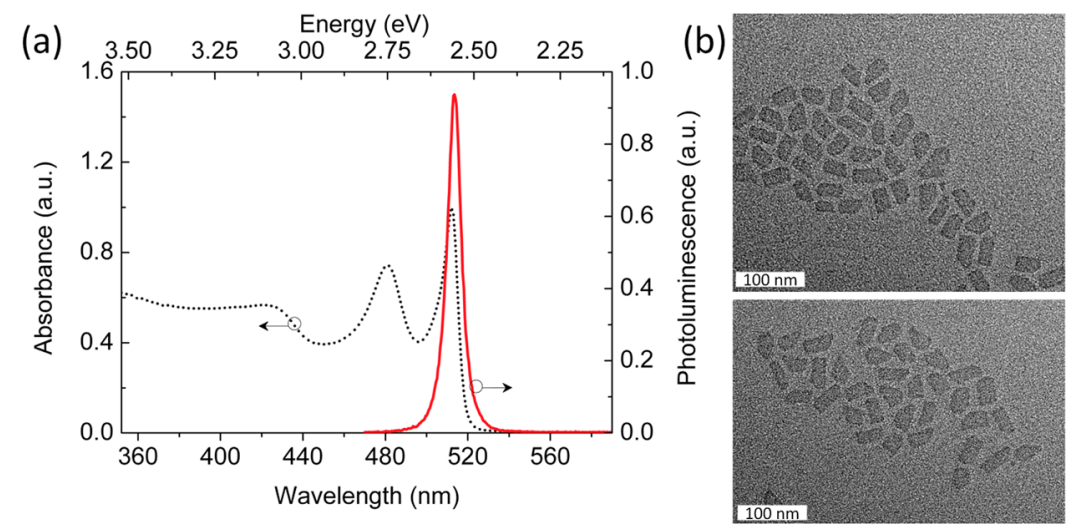

Figure 1. (a) Absorbance (dashed line) and photoluminescence (solid line) spectrum; (b) transmission electron microscopy images of the $4 \mathrm{ML}$ CdSe NPLs.

in the stacked NPLs. ${ }^{20}$ Additionally, polarized light emission was demonstrated in the stacked NPL assemblies. ${ }^{19}$ However, steady-state and transient excitonic properties of the stacked NPLs have not been understood nor elucidated to date. Moreover, strong near-field dipole-dipole coupling between the NPLs of the same population in their close-packed stacked assemblies has not been considered before. Here, we hypothesize that excitons should efficiently hop back and forth between the NPLs within the stacked assemblies via resonant nonradiative energy transfer, also known as Förster resonance energy transfer (FRET). ${ }^{21}$ In the case of colloidal quantum dot (QD)-based closepacked assemblies, FRET was observed within the same population of the QDs, resulting in a red-shift of the photoluminescence spectrum due to the intrinsic inhomogeneous size broadening favoring downhill exciton funneling toward QDs having a smaller band gap. ${ }^{22,23}$ In the case of NPLs, the density of states is not inhomogeneously broadened since the magic-sized vertical thickness (e.g., 3, 4, or 5 monolayers) of the NPLs dictates their band gap energy. ${ }^{15,17,25}$ Quantum confinement due to the lateral direction in the NPLs is very weak because lateral sizes are typically larger than the exciton Bohr radius. ${ }^{15}$ Therefore, one should expect to observe different signatures for the exciton transfer via FRET in stacked NPL assemblies as compared to ones in close-packed QD assemblies.

In this work, we systematically investigate and report the steady-state and transient excitonic properties of controlled stacked NPL assemblies and reveal ultralong exciton migration in stacked NPL assemblies. Controlled stacking of the CdSe NPLs having a 4 monolayer vertical thickness is achieved in solution phase via stepby-step addition of ethanol to the solution. Transmission electron microscopy imaging supports the controlled formation of the NPL stacks. An ultralong Förster radius of $\sim 13.5 \mathrm{~nm}$ and extremely long exciton migration length of $133 \mathrm{~nm}$ are found for the stacked NPLs thanks to close-packed stacking, strong selfspectral overlap arising due to the large extinction coefficient of the NPLs, and the parallel and collinear orientation of the transition dipoles in column-like ordered NPL stacks. However, in the NPL stacks, we do not observe the common signatures of homo-FRET: red-shifting photoluminescence emission as observed in the close-packed assemblies of the colloidal nanocrystals. This is because of the fact that there is no inhomogeneous broadening in the NPLs. We observe that as the NPLs form into large stacks, the photoluminescence quantum yield (QY) of the NPLs decreases by a factor as high as 10-fold and transient fluorescence decay of the NPLs is also concomitantly accelerated. These observations are accounted for trapping of the excitons by nonemissive NPLs in the stacked NPL assemblies assisted by homo-FRET (i.e., FRET in between the same populations of emitters) within the stacks. Trapped excitons then undergo rapid nonradiative recombination, accelerating the overall photoluminescence decay kinetics. We develop a rateequation-based model that considers the possible excitonic interactions (i.e., the exciton transfer and the radiative and nonradiative recombination) and the actual distribution of the stack size using TEM imaging. The results of the model are in excellent agreement with the experimental time-resolved fluorescence results. Consequently, we reveal the critical significance of stacking control in NPL solids for their potential use in optoelectronics.

\section{RESULTS AND DISCUSSION}

CdSe NPLs having a vertical thickness of $1.2 \mathrm{~nm}$ corresponding to 4 monolayers (MLs) of CdSe lattice unit are synthesized using a modified method and dissolved in hexane (see the Experimental Section). ${ }^{8}$ Figure 1a shows the absorbance (dashed line) and photoluminescence (solid line) spectrum of the $4 \mathrm{ML}$ CdSe NPLs in solution. After size-selective precipitation via ultracentrifugation, there are no side-products such as colloidal quantum dots or different vertical thickness NPLs, as can be seen by the pure 4 ML NPL emission and absorbance spectra in Figure 1a. Characteristic of 
(a)

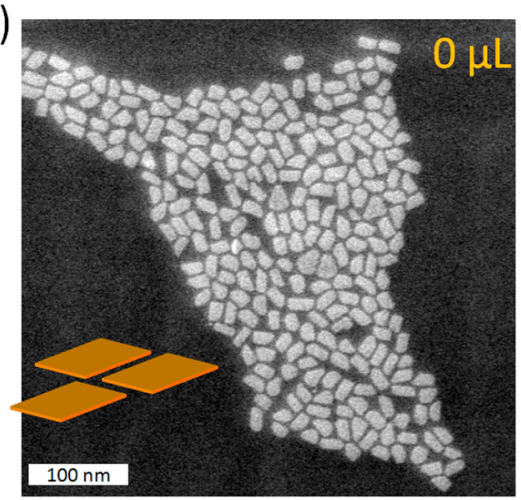

(c)

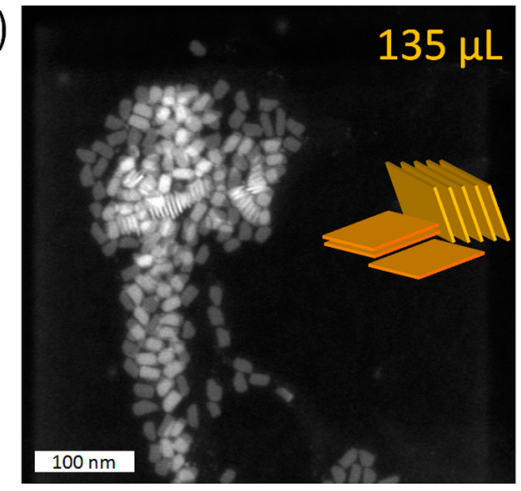

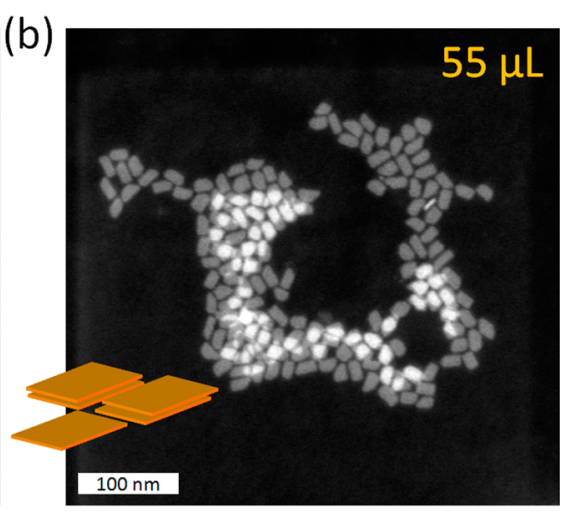

(d)

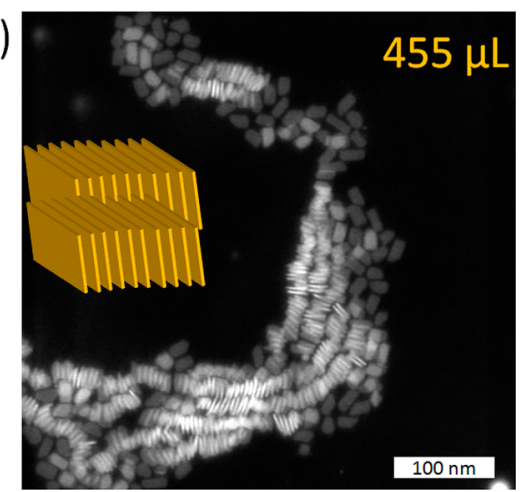

Figure 2. High-angle annular dark-field (HAADF) TEM images of the NPLs with (a) no ethanol and a total of (b) $55 \mu \mathrm{L}$, (c) $135 \mu \mathrm{L}$, and (d) $455 \mu \mathrm{L}$ of ethanol added. The scale bars are $100 \mathrm{~nm}$. As the total added ethanol amount is increased gradually, longer column-like stacks of the NPLs are formed. On each image, a cartoon-like depiction of the evolution of the stacking in NPLs is presented by yellow-colored NPLs.

the colloidal NPLs, we observe an absorbance spectrum featuring two peaks, at 480 and $512 \mathrm{~nm}$, corresponding to the electron/light-hole and electron/ heavy-hole transitions, respectively. The photoluminescence emission peak is located at $513 \mathrm{~nm}$ with a fwhm of $\sim 8 \mathrm{~nm}$. Transmission electron microscopy (TEM) images of the 4 ML NPLs are shown in Figure 1b, exhibiting a rectangular shape with an average lateral size of $20.08 \mathrm{~nm}( \pm 2.36 \mathrm{~nm})$ by $11.50 \mathrm{~nm}( \pm 1.39 \mathrm{~nm})$. Therefore, quantum confinement along lateral dimensions is expected to be very weak in these NPLs since the lateral size is much larger than the size $(\sim 5-7 \mathrm{~nm})$ of the first exciton. ${ }^{15,25}$

Previously, Dubertret et al. demonstrated that addition of a polar solvent such as ethanol into the solution of the NPLs, which is dissolved in apolar solvents (e.g., hexane and toluene), stacks the NPLs on top of each other, resulting in column-like assemblies. ${ }^{19}$ When ethanol is introduced, NPLs tend to minimize their surface energy by drawing away ethanol since ethanol is an antisolvent for the ligands (oleic acid) of the NPLs. Removal of the oleic acid ligands due to ethanol was shown previously. ${ }^{20,26}$ Therefore, addition of ethanol tends to bring NPLs together, where strong van der Waals forces between the surfaces of the NPLs induce the formation of column-like 1D stacks of the NPLs (see Figure S1). We observe that, once stacked, NPLs cannot be separated anymore due to strong attractive forces.
Previously, small-angle X-ray scattering (SAXS) measurements confirmed the formation of the ordered structures with a spatial period of $\sim 5 \mathrm{~nm} .{ }^{20}$ Here, we find the average center-to-center distance between the stacked NPLs to be $4.29 \mathrm{~nm}( \pm 0.36 \mathrm{~nm})$ using highresolution TEM imaging (see Figure $\mathrm{S1}$ ).

To initiate and control the degree of stacking, we added ethanol carefully step by step to the solution of the $4 \mathrm{ML}$ NPLs (in a total of $3.1 \mathrm{~mL}$ volume having $16.79 \mathrm{nM}$ of the NPLs). We could sensitively control the degree of stacking via tuning the amount of added ethanol to the solution of the NPLs. Figure 2 exhibits the high-angle annular dark-field (HAADF) TEM images of NPLs for different amounts of ethanol addition $(0,55$, 135 , and $455 \mu \mathrm{L}$ of ethanol added in total in the corresponding samples of Figure 2a to d, respectively). When there is no ethanol added to the solution of the NPLs, we could not detect any discernible stacking. Figure 2a exhibits completely flat-lying NPLs without any stacking. Figure $2 \mathrm{~b}$ shows the NPLs when $55 \mu \mathrm{L}$ of ethanol has been added to the solution. In this case, we can clearly observe that stacking has been initiated. The contrast difference between the NPLs in the TEM image reveals the presence of two NPLs sitting on top of each other (i.e., brighter) or nonstacked NPLs (i.e., darker). However, most of the NPLs are still nonstacked. As we further continued adding ethanol, as shown in Figure $2 \mathrm{c}$ for a total of $135 \mu \mathrm{L}$ added ethanol, we start to 
(a)

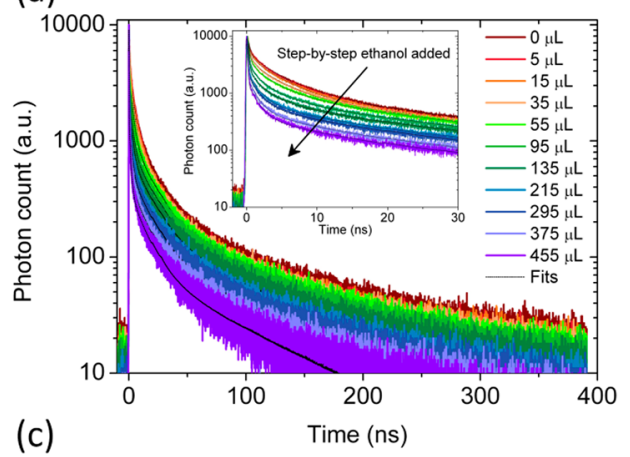

(c)

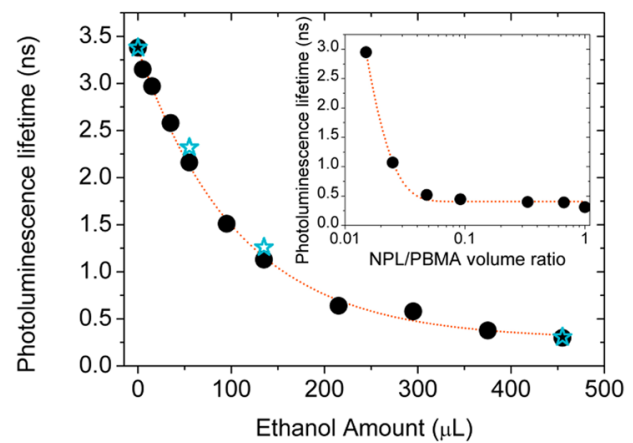

(b)

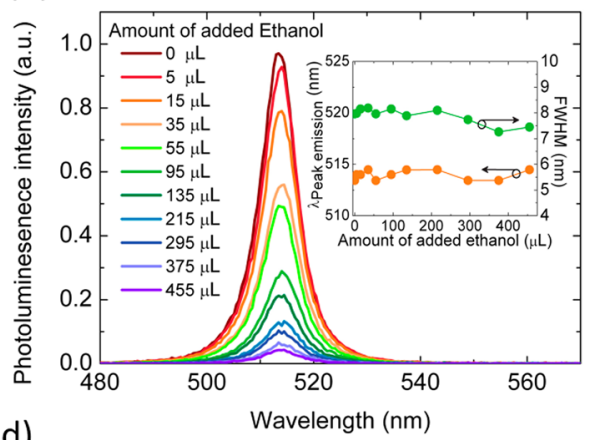

(d)

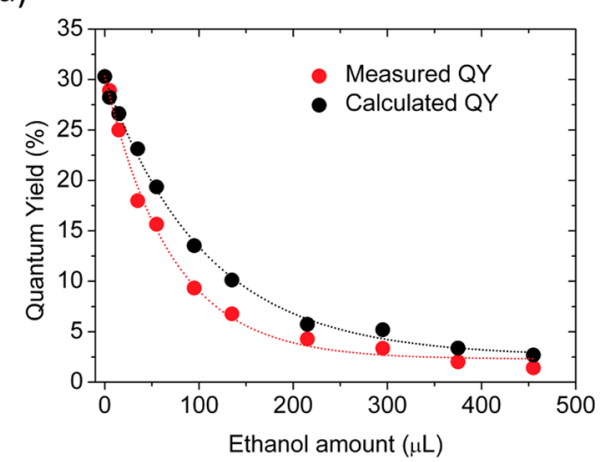

Figure 3. (a) Time-resolved photoluminescence decays together with their multiexponential fits. Inset shows the same photoluminescence decay curves in a shorter time window. (b) Steady-state photoluminescence intensity of the NPLs while ethanol is added gradually to the solution of the NPLs. Inset shows the peak emission wavelength and photoluminescence fwhm of the NPLs as a function of the total added amount of ethanol. (c) Amplitude-averaged photoluminescence lifetimes of the NPLs shown by black dots as a function of the total added ethanol amount. Blue stars show the modeled photoluminescence lifetimes of the NPLs for $0,55,135$, and $455 \mu \mathrm{L}$ of ethanol added cases. The inset shows the average photoluminescence lifetimes of the NPLs in PBMA host matrix given for different NPL/PBMA volume ratios. Transition from nonstacking to stacking in the photoluminescence decay kinetics of the NPLs is observed without using any ethanol. As the NPL/PBMA volume ratio is gradually increased, phase segregation occurs that results in stacking. This increases the total decay rate of the NPLs. (d) Photoluminescence QY of the NPLs as a function of total added ethanol amount measured directly from steady-state photoluminescence measurements and calculated semiempirically from the photoluminescence lifetimes assuming the radiative recombination does not change.

observe the formation of the flat-lying stacks of $2-3$ layers of NPLs on top of each other. Also we observe longer stacks (more than 4 nanoplatelets stacked together) lying perpendicular to the TEM grid. Finally, the NPLs mostly form column-like ordered longer stacks when a total of $455 \mu \mathrm{L}$ of ethanol was added to the solution, as shown in Figure $2 \mathrm{~d}$.

We now investigate the optical properties of the stacked NPL assemblies via steady-state and transient photoluminescence spectroscopy. Figure 3a exhibits the measured photoluminescence decay curves and their fits. As the total added amount of ethanol is increased ( 0 to $455 \mu \mathrm{L}$ ), photoluminescence decays become considerably faster. The inset of Figure 3 a depicts the shorter time window of the decay curves clearly exhibiting the acceleration of the fast decay components. Figure $3 \mathrm{~b}$ presents the steady-state photoluminescence spectra of the same samples having different amounts of ethanol added to the solution. The photoluminescence intensity quickly drops as stacking is initiated. Quenching of the photoluminescence intensity is found to be as high as 10 -fold when a total of $455 \mu \mathrm{L}$ of ethanol is used for a high degree of stacking.
These observed features may indicate the presence of exciton migration in these NPL stacks via homoFRET. Previously, in close-packed QD solids, homoFRET has been shown to possess characteristic signatures including strong red-shift of the emission spectrum due to downhill funneling of the excitons within the inhomogeneously broadened density of states in the QDs. Furthermore, exciton transfer within solids of the same QD population was shown to decrease photoluminescence quantum yield due to trapping by small band gap and nonemissive QDs. ${ }^{27,28}$ Finally, homo-FRET in the energetically disordered QD populations leads to strong modification (shortening) of the photoluminescence lifetimes at the blue-tail emission of the QDs. ${ }^{24,29-32}$ However, in the stacked NPL assemblies contrary to the QDs, we observe neither a shift in the peak emission wavelength (see Figure $3 \mathrm{~b}$ and its inset) nor a significant modification in the fluorescence lifetimes at different spectral positions of the emission (see Figure S2). In the NPLs, downhill migration of the excitons (i.e., red-shift) would not be expected since all of the NPLs have the same magicsized (4 ML in this case) vertical thickness; thus, there is 
no inhomogeneous broadening due to size. Furthermore, fwhm of the NPL emission is observed not to change in a significant way with the stacking (see Figure $3 b$ inset). This indicates that the ambient dielectric environment around the transition dipole in an NPL also does not change significantly as they form into stacks. This may be due to the fact that the inorganic crystal part of the NPLs, which has a larger dielectric constant, is thinner as compared to the bulky organic ligands surrounding the NPLs, which have a lower dielectric constant. Therefore, the dielectric constant of the ligands would largely set the average dielectric constant within the NPL stacks. These observations imply that NPLs having no inhomogeneous broadening, strongly contrasting with common colloidal semiconductor nanomaterials, do not exhibit the widely known signatures of the exciton transport/ migration in their steady-state and transient photoluminescence properties.

In Figure 3a photoluminescence decay curves are fitted using four exponential decay functions after deconvolving with the instrument response function of the time-resolved fluorescence system (see the Experimental Section). Multiexponential decay (three or four exponential decay functions) in the NPLs was also previously observed., ${ }^{9,26}$ Figure $3 c$ depicts the amplitude-averaged photoluminescence lifetimes of the NPLs as a function of total added ethanol amount (see Figure S3 for the individual lifetime components). The NPLs have a $\sim 3.38 \mathrm{~ns}$ average photoluminescence lifetime before stacking is induced, which is in accordance with the photoluminescence lifetime of the $4 \mathrm{ML}$ CdSe NPLs. ${ }^{9}$ As ethanol is added gradually to the solution of the NPLs, we observe that photoluminescence lifetime of the NPLs can decrease as much as by an order of magnitude. We observe the fastest amplitude-averaged photoluminescence lifetime (300 ps) in the case of a total $455 \mu \mathrm{L}$ of ethanol added to the NPL solution, where the longest stacks are present. Before inducing any stacking, the photoluminescence QY is measured to be $30.3 \%$ by comparing the emission of the NPLs to a reference dye of rhodamine $6 \mathrm{G}$ having a quantum yield of $95 \%$. Figure $3 \mathrm{~d}$ (red dots) demonstrates the change of the measured photoluminescence QYs as a function of total added ethanol amount, where the QY decreases in a similar trend to that observed for the photoluminescence lifetimes. In the case of $455 \mu \mathrm{L}$ of ethanol added to the NPL solution, the QY is measured to be as low as $1.42 \%$.

To understand the underpinning physics of decreased photoluminescence QYs in the stacked NPLs, we correlate the measured photoluminescence QYs to the amplitude-averaged photoluminescence lifetimes as shown in Figure 3c. From QY $=\left(\gamma_{\text {radiative }} / \gamma_{\text {total }}\right)$, we calculate $\gamma_{\text {radiative }} \approx 0.090 \mathrm{~ns}^{-1}$ when no ethanol is added. Then, we obtain the photoluminescence QYs as a function of the added ethanol amount utilizing the measured amplitude-averaged photoluminescence lifetimes $\left(\gamma_{\text {total }}=1 / \tau_{\text {total }}\right)$ and assuming that the radiative rate $\left(\gamma_{\text {radiative }}\right)$ does not change upon stacking. Except for the change of the effective dielectric medium, radiative recombination rate is not expected to change upon stacking as observed for the other nanoemitters. ${ }^{33}$ The calculated photoluminescence QYs are given in Figure 3d (black dots). These two QYs (i.e., measured and calculated) are in a good agreement with each other. This supports that the radiative rate $\left(\gamma_{\text {radiative }}\right)$ does not change upon stacking of the NPLs, whereas the nonradiative recombination rate of the excitons significantly increases. Our hypothesis is that stacking of the NPLs should lead to efficient exciton migration among the NPLs via homo-FRET, similar to the close-packed QD solids. The exciton transport via homo-FRET in the NPL stacks strongly assists trapping of the excitons by nonemissive NPLs (i.e., defected NPLs) existing within the same population. Therefore, the nonradiative recombination channels are effectively boosted in the stacked NPL assemblies.

Addition of ethanol to the solution of the NPLs may also cause removal of the surface ligands of the NPLs, which might create a larger number of nonemissive NPLs in the population. ${ }^{26,34-36}$ To eliminate the possibility that addition of ethanol to the NPL solution may cause any significant effect on the decay kinetics of the NPLs, we test stacking of the NPLs without using ethanol. To this end, we incorporate the same NPLs (with a concentration of $517.61 \mathrm{nM}$ ) into PBMA (poly(butyl methacrylate), 1 wt \%) insulating the host polymer matrix at varying NPL/PBMA volume ratios and investigate the transient photoluminescence kinetics in the drop-casted solid film samples. It has been also known that solid film formation can trigger the stacking of the NPLs due to solvent evaporation. ${ }^{19}$ In addition, phase segregation between the colloidal nanocrystals and the polymers is known to increase the aggregation of the colloidal nanocrystals, which would possibly cause the stacking of the NPLs. ${ }^{37-39} \mathrm{We}$ present the amplitude-averaged photoluminescence lifetimes of the NPLs in these different solid film samples as shown in the inset of Figure $3 c$ (also see the decay curves in Figure S4). First, by using a very low density of NPLs in the polymer matrix, we intentionally prevented stacking of the NPLs via suppressing phase segregation. Therefore, the photoluminescence lifetime in the sample that has the smallest NPL/PBMA volume ratio $(\sim 0.015)$ is $\sim 3 \mathrm{~ns}$, which is in very good agreement with that ( $\sim 3.38 \mathrm{~ns})$ of the nonstacked NPLs in solution phase before adding any ethanol (see Figure 3c). This strongly suggests that the NPLs are well separated in the host matrix at small NPL/PBMA volume ratios. As the NPL/PBMA volume ratio is increased from 0.025 to 1 , the average photoluminescence lifetime of the NPLs decreases from $\sim 1$ to $\sim 0.3$ ns, respectively (see Figure $3 c$, inset). Therefore, 
the lifespan ( 3 to $0.3 \mathrm{~ns}$ ) is successfully realized using the NPL:PBMA samples exhibiting an excellent quantitative agreement with the photoluminescence lifetimes (3.38-0.3 ns) of the nonstacking and stacking NPLs in solution phase. Consequently, we can observe the transition from nonstacking to stacking in the photoluminescence decay kinetics of the NPLs in the NPL:PBMA samples without using any ethanol. This observation also strongly suggests that stacking is the main cause of the stark changes observed in the photoluminescence decay kinetics of the NPLs.

Previously, increased exciton decay rates and quenched photoluminescence intensity were observed in dense fluorescent dye solutions. ${ }^{40,41}$ This was attributed to the exciton hopping among dye chromophores and quenching via nonemissive dye dimers. Theoretically, Loring, Anderson, and Fayer developed a model of homo-FRET systems and showed that these systems are prone to strong quenching due to trapping. ${ }^{42}$ Also in the stacked NPL assemblies, excitons are expected to hop back and forth between the NPLs along the column-like ordered stacked assembly due to strong near-field dipole-dipole coupling between the NPLs. There is no significant Stokes shift $(\sim 1 \mathrm{~nm})$ in the NPLs enabling a large self-overlap between the emission and absorption spectra. Here, for the NPLs in the same stack, we find the Förster radius $\left(R_{0}\right),{ }^{21,43}$ the separation distance at which the exciton transfer efficiency is $50 \%$, to be $13.48 \mathrm{~nm}$. In the calculation of the Förster radius the dipole orientation term $\left(\kappa^{2}\right)$ is generally assumed to be $2 / 3$ due to dynamic averaging of the dipoles that are randomized by the rotational diffusion in solution. In the case of column-like oriented stacks of the NPLs, transition dipoles are expected to be both parallel and collinear. Therefore, here, the $\kappa^{2}$ term can take a maximum value of $4 .{ }^{43}$ Other parameters used to predict the Förster radius are the photoluminescence QY of the NPLs of $30 \%$, the refractive index $(n)$ of 1.8 , and the extinction coefficient of $3.1 \times 10^{-14} \mathrm{~cm}^{2}$ at $400 \mathrm{~nm}$ for the $4 \mathrm{ML}$ CdSe NPLs. ${ }^{17}$ When stacked, the center-to-center distance between the NPLs has been measured to be $4.29 \mathrm{~nm}$ using high-resolution TEM imaging (considering a $1.2 \mathrm{~nm}$ thickness of the NPLs, oleic acid ligands have to be interpenetrating). ${ }^{20,29}$ Overall, FRET efficiency is calculated to be as high as $99.9 \%$ for the exciton transfer between neighboring NPLs within the stacked assemblies. NPL-to-NPL hopping time can be as fast as $\sim 3$ ps. Therefore, excitons can hop many times among the NPLs within the stacks before either radiative or nonradiative recombination occurs. In thin film organic semiconductors, Menke et al. ${ }^{44}$ described the exciton migration length $\left(L_{D}\right)$ assuming isotropic transport using

$$
L_{D}=\sqrt{A} \frac{R_{0}{ }^{3}}{d^{2}}
$$

where $R_{0}$ is the Förster radius, $d$ is the center-to-center distance between the donor-acceptor species, and $A$ is a term related to distribution of the molecular separation in the amorphous film, which is generally close to $1 .{ }^{44}$ Using eq 1 , the calculated exciton migration length is found to be as high as $133.1 \mathrm{~nm}$ $( \pm 12.0 \mathrm{~nm}$ ) with the assumption of isotropic transport giving us a lower limit for the exciton migration length in the stacks of the NPLs. This exciton migration length represents the longest one among all other colloidal semiconductor nanocrystals reported to date. ${ }^{32}$ Therefore, this suggests that stacked NPL assemblies are promising for light-harvesting applications owing to their ultra-long-range exciton migration capability.

In the presence of ultra-long-range exciton migration in the NPL stacks, quenching of the photoluminescence emission is explained by exciton-migrationassisted exciton sinking in the stacked NPLs. Figure 4 schematically highlights this exciton quenching mechanism. In the case of nonstacked NPLs, exciton recombination has its own intrinsic radiative and nonradiative channels. In the case of stacked NPLs, ultraefficient homo-FRET in the absence of inhomogeneous broadening should not have increased the intrinsic decay rates in the NPLs unless there are excitontrapping nonemissive NPLs. In CdSe-based nanocrystals it is well known that surface defects, poorly passivated surface sites, and crystal defects could cause various trapping mechanisms, resulting in nonemissive nanocrystals. ${ }^{45-48}$ Hole trapping in CdSe nanocrystals was found to be related to poor surface passivation and $\mathrm{Cd}$ vacancies ${ }^{36}$ and was shown to cause fast nonradiative recombination of the excitons with time constants on the order of 10 ps. ${ }^{49,50}$ In the CdSe NPLs, it is expected that there might arise both surface traps and $\mathrm{Cd}$ vacancies, leading to interband hole trap states, which may rapidly quench the excitons nonradiatively. ${ }^{15}$ Previously, blinking was also reported in the NPLs, suggesting that nonemissive or dark states arise in the NPL populations. ${ }^{25,26}$ Here in Figure 4, a nonemissive NPL (red color) is considered to act as an exciton sink and to quench the emission of the other NPLs (yellow color) in the stack due to long-range exciton transfer boosting the trapping. As the stacked assemblies grow larger, more of the NPLs are affected by the trapping since the ultralong exciton transfer range of $130 \mathrm{~nm}$ spans the entire or majority of the stack. Therefore, the presence of even a few nonemissive NPLs can strongly quench the photoluminescence intensity from a long NPL stack. Synthesis of NPLs for light-generation applications in the future would require minimizing the density of the nonemissive NPLs for achieving high quantum yield.

To develop a deeper understanding and explain the evolution of the photoluminescence decay kinetics of the NPLs as they form into stacks, we also developed a rate equation model that accounts for the excitonic 

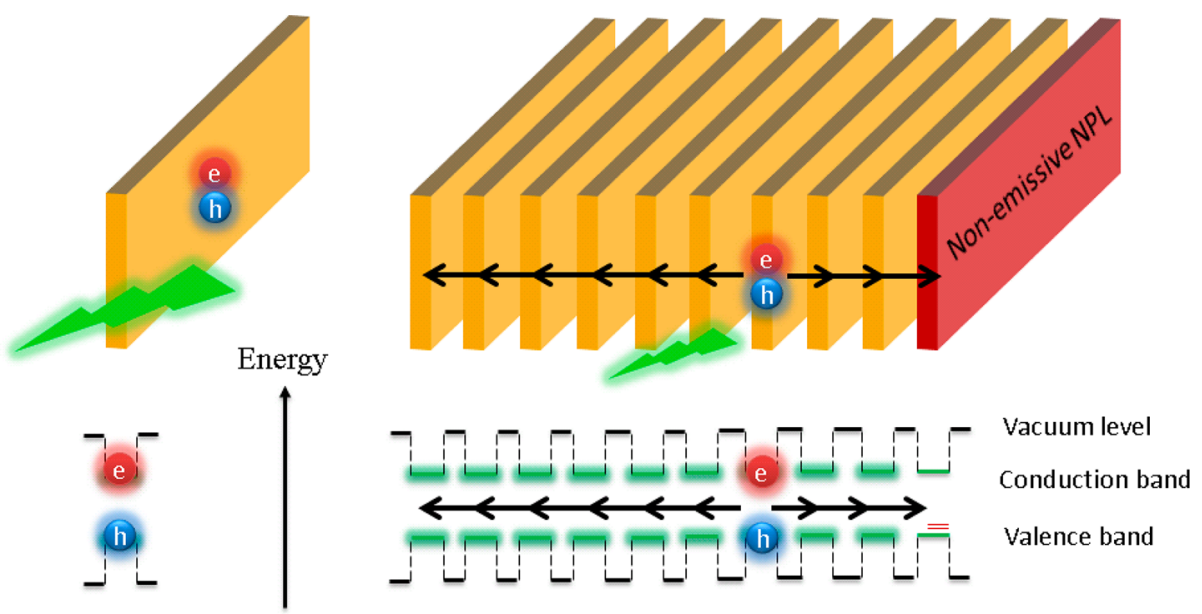

Exciton transfer via homo-FRET

Trapping

Figure 4. Schematic depicting the ultra-long-range exciton transfer in the stacked NPL assemblies via exciton hopping through homo-FRET. The nonemissive NPL in the stack causes trapping with the assistance of long-range exciton migration, causing nonradiative recombination of the excitons, which quenches the photoluminescence emission of the NPLs in the stack.

interactions in the stacked NPLs: the exciton transfer among the NPLs, the radiative recombination in the NPLs, and the fast nonradiative recombination (i.e., hole trapping) at the defected NPLs. In the model, we consider the size distribution of the stacks (see Figure S6) as investigated via TEM imaging for three different ethanol-added cases (i.e., 55, 135, and $455 \mu \mathrm{L}$ ). To construct the rate equations, we consider a stack with a fixed size (i.e., fixed number of NPLs), say $k$. In this stack, $m$ of these NPLs are taken to be defected ( $m \leq k$ ) depending on the fraction of the defected NPL population. Here, let $n_{i}$ denote the number of the ith NPL $(i=1: k)$ that is excited. The rate equations are then constructed for each NPL within the stack of size $k$ as

$$
\begin{gathered}
\frac{\mathrm{d} n_{1}}{\mathrm{~d} t}=-\left(\gamma_{1}+\gamma_{\mathrm{tr}}\right) n_{1}+\gamma_{\mathrm{tr}} n_{2} \\
\frac{\mathrm{d} n_{2}}{\mathrm{~d} t}=-\left(\gamma_{2}+2 \gamma_{\mathrm{tr}}\right) n_{2}+\gamma_{\mathrm{tr}}\left(n_{1}+n_{3}\right) \\
\vdots \\
\frac{\mathrm{d} n_{k}-1}{\mathrm{~d} t}=-\left(\gamma_{k-1}+2 \gamma_{\mathrm{tr}}\right) n_{k-1}+\gamma_{\mathrm{tr}}\left(n_{k}-2+n_{k}\right) \\
\frac{\mathrm{d} n_{k}}{\mathrm{~d} t}=-\left(\gamma_{k}+\gamma_{\mathrm{tr}}\right) n_{k}+\gamma_{\mathrm{tr}} n_{k-1}
\end{gathered}
$$

Here, $\gamma_{i}$ is the recombination rate associated with an NPL located in the ith position of the stack and is defined as

$$
\gamma_{i}=\left\{\begin{array}{c}
\gamma+\gamma^{\prime}, i=d_{1}, d_{2}, \ldots, \text { or } d_{m} \\
\gamma, \text { otherwise }
\end{array}\right.
$$

where $\gamma$ is the effective recombination rate of the nondefected NPLs (including the radiative and the intrinsic nonradiative recombination in the nondefected NPLs). $\gamma^{\prime}$ is the nonradiative recombination rate in a defected NPL due to fast hole trapping. The defected NPLs can be at the positions $d_{1}, d_{2}, \ldots, d_{m}$, where $d_{i}$ 's refer to the locations of NPLs in the stack depending on the fraction of the defected NPL population. $\gamma_{\mathrm{tr}}$ is the exciton transfer rate between two consecutive NPLs within the same stack. These transfer and recombination channels are schematically illustrated in Figure S5 for a representative stack. The exciton transfer rate $\left(\gamma_{\mathrm{tr}}\right)$ is $(3 \mathrm{ps})^{-1}$ as calculated using the Förster radius and the inter-NPL separation within the stacks, the nonradiative recombination rate $\left(\gamma^{\prime}\right)$ at the trap sites in defected NPLs is $(35 \mathrm{ps})^{-1}$ due to fast hole trapping, ${ }^{15}$ and the effective recombination rate $(\gamma)$ is $(3.38 \mathrm{~ns})^{-1}$.

The last parameter to determine is the fraction of the defected NPL population $(r)$ within the whole NPL population. Depending on the synthesis route, the NPL populations may include NPLs having hole-trap sites. A recent report has also revealed that a varying fraction of the CdSe NPLs may contain hole traps as observed by time-resolved fluorescence and pumpprobe transient absorption measurements. ${ }^{51}$ To find the percentage of the defected NPL population $(r)$, we swept this parameter to match the modeled lifetime with the experimental lifetime of the $55 \mu \mathrm{L}$ of ethanol added case. We chose the $55 \mu \mathrm{L}$ of ethanol added case due to its simple stack size distribution consisting of stacks of either only one or two nanoplatelets (see Figure S6). The resulting fraction of the defected NPL population is found to be $r=0.22$. We use the same defected NPL population fraction for all three cases (i.e., $55,135$, and $455 \mu \mathrm{L})$.

Using the model we calculate the photoluminescence lifetimes (see Supporting Information for the details) of the stacked NPL ensembles in 55, 135, and $455 \mu \mathrm{L}$ of ethanol added cases as $2.32,1.26$, and 0.306 ns, respectively. These calculated lifetimes are shown in Figure $3 c$ with blue stars together with the no ethanol added case as a trivial solution. The lifetimes calculated by the model exhibit an excellent agreement with the experimental lifetimes for the three ethanol-added cases of 55,135 , and $455 \mu \mathrm{L}$, which were measured to be $2.16,1.13$, and $0.300 \mathrm{~ns}$, 
respectively. The error between the modeled lifetimes and the experimental lifetimes is less than $12 \%$. This excellent agreement between the model and the experiment in three different stacked NPL ensembles exhibits vigorous support for the hypothesis that homo-FRET-assisted exciton trapping and nonradiative recombination are the dominant excitonic effects in the stacked NPLs. Furthermore, the fraction of the defected NPL population ( $r=0.22$ ) works very well for all three cases, and this elucidates that adding ethanol does not cause additional nonradiative trap sites. Moreover, using the model, we can infer new information on the decay kinetics of the stacked NPLs. Figure S8 shows the photoluminescence lifetime of a hypothetical stack of a fixed size (i.e., 35) while varying the position of a single defected NPL within this stack. We observe that the position of the defected NPL is also important for the exciton trapping. For a defected NPL in the middle of the stack, the trapping efficiency is larger as compared to the case where the defected NPL is in the tail of the stack.

As an alternative mechanism to exciton hopping via FRET, radiative energy transfer (RET) between the NPLs in the stacks is possible. RET is difficult to distinguish from the FRET in steady-state measurements. ${ }^{52}$ The small difference between the calculated and experimental quantum yields (see Figure $3 \mathrm{~d}$ ) could probably be explained as due to the presence of RET. However, investigation of the transient photoluminescence kinetics and modeling as a function of controlled stacking strongly suggests that the dominant exciton transport mechanism is based on nonradiative exciton transfer (i.e., homo-FRET). RET, alternatively, can support the transport of the excitons among the different stacks of the NPLs since FRET would not be efficient among the NPLs within different NPL stacks due to large lateral sizes (i.e., $10 \mathrm{~nm}$ or larger) limiting the exciton hopping between different stacks.

\section{CONCLUSIONS}

In summary, we have demonstrated controlled stacking of the NPLs that exhibit different steady-state and transient excitonic properties as compared to the nonstacked NPLs. First, photoluminescence quantum yield and lifetime are decreased by an order of magnitude when long column-like stacks of the NPLs are formed. These observations are well explained by the ultralong exciton transfer among the NPLs within the stacked assembly owing to ultraefficient homo-FRET. However, exciton transport increases trapping of the excitons by nonemissive NPLs, acting as exciton sinks. This causes the strong quenching of the photoluminescence and acceleration of the decay rates. The exciton transfer in the NPLs exhibits different signatures as compared to the colloidal quantum dots due to the absence of inhomogeneous broadening in the NPLs. These observations include no red-shift and fixed emission line-width despite ultraefficient exciton transfer. Moreover, we developed a rate-equation-based model that strongly agrees with the observed changes in the photoluminescence decay kinetics explained by ultraefficient exciton-transfer-assisted exciton trapping in the NPL stacks due to the presence of defected NPLs. Overall, the results show the critical significance of stacking control in NPL solids for efficient light generation and harvesting.

\section{EXPERIMENTAL SECTION}

Synthesis of the 4 ML CdSe NPLs. For a typical synthesis, $170 \mathrm{mg}$ of cadmium myristate, $12 \mathrm{mg}$ of selenium, and $15 \mathrm{~mL}$ of octadecene (ODE) are loaded into a three-neck flask. After evacuation of the solution at room temperature, the solution is heated to $240{ }^{\circ} \mathrm{C}$ under an argon atmosphere. When the temperature reaches $195^{\circ} \mathrm{C}$, the color of the solution becomes yellowish. Then, $45 \mathrm{mg}$ of cadmium acetate dihaydrate is introduced. After 4 min of growth of CdSe NPLs at $240{ }^{\circ} \mathrm{C}$, the reaction is stopped and cooled to room temperature with the injection of $1 \mathrm{~mL}$ of oleic acid (OA). Four ML CdSe NPLs are separated from other reaction products with the successive purification steps.

Time-Resolved Photoluminescence Spectroscopy. Pico Quant FluoTime 200 equipped with a TimeHarp time-correlated singlephoton counting (TCSPC) unit is utilized to record the fluorescence decays with a $375 \mathrm{~nm}$ picosecond pulse laser. The fullwidth at half-maxima of the instrument response function (IRF) is $200 \mathrm{ps}$. Per-pulse energy of the pump laser was measured to be $15.6 \mathrm{pJ}$ at $3.31 \mathrm{eV}$ (spot radius is $\sim 100 \mu \mathrm{m}$ ). The FluoFit program is employed for deconvolution fitting with the IRF. Fourth-order exponential decay models are employed to fit the photoluminescence decay curves of the NPLs, resulting in a reduced $\chi^{2}$ of $\sim 1$ and uniform residuals.

Conflict of Interest: The authors declare no competing financial interest.
Supporting Information Available: TEM of the highly stacked NPLs, analysis of the multiexponential photoluminescence lifetime and photoluminescence decays of the NPL:PBMA samples, and rate-equation-based model for the exciton migration and nonradiative recombination in the NPL stacks. This material is available free of charge via the Internet at http://pubs.acs.org.

Acknowledgment. The authors would like to acknowledge the financial support from EU-FP7 Nanophotonics4Energy NoE, TUBITAK EEEAG 109E002, 109E004, 110E010, 110E217, NRF-RF2009-09, NRF-CRP-6-2010-02, and A*STAR of Singapore. H.V.D. acknowledges support from ESF-EURYI and TUBA-GEBIP.

\section{REFERENCES AND NOTES}

1. Murray, C. B.; Norris, D. J.; Bawendi, M. G. Synthesis and Characterization of Nearly Monodisperse CdE (E = Sulfur, Selenium, Tellurium) Semiconductor Nanocrystallites. J. Am. Chem. Soc. 1993, 115, 8706-8715.

2. Peng, X.; Manna, L.; Yang, W.; Wickham, J.; Scher, E.; Kadavanich, A.; Alivisatos, A. Shape Control of CdSe Nanocrystals. Nature 2000, 404, 59-61.

3. Talapin, D. V.; Lee, J.-S.; Kovalenko, M. V.; Shevchenko, E. V. Prospects of Colloidal Nanocrystals for Electronic and Optoelectronic Applications. Chem. Rev. 2010, 110, 389-458. 
4. Talapin, D. V.; Nelson, J. H.; Shevchenko, E. V.; Aloni, S.; Sadtler, B.; Alivisatos, A. P. Seeded Growth of Highly Luminescent $\mathrm{CdSe} / \mathrm{CdS}$ Nanoheterostructures with Rod and Tetrapod Morphologies. Nano Lett. 2007, 7, 29512959.

5. Milliron, D. J.; Hughes, S. M.; Cui, Y.; Manna, L.; Li, J.; Wang, L.-W.; Alivisatos, A. P. Colloidal Nanocrystal Heterostructures with Linear and Branched Topology. Nature 2004, 430, 190-195.

6. Park, J.; An, K.; Hwang, Y.; Park, J.-G.; Noh, H.-J.; Kim, J.-Y.; Park, J.-H.; Hwang, N.-M.; Hyeon, T. Ultra-Large-Scale Syntheses of Monodisperse Nanocrystals. Nat. Mater. 2004, 3, 891-895.

7. Hines, M. A.; Guyot-Sionnest, P. Synthesis and Characterization of Strongly Luminescing ZnS-Capped CdSe Nanocrystals. J. Phys. Chem. 1996, 100, 468-471.

8. Ithurria, S.; Dubertret, B. Quasi 2D Colloidal CdSe Platelets with Thicknesses Controlled at the Atomic Level. J. Am. Chem. Soc. 2008, 130, 16504-16505.

9. Ithurria, S.; Tessier, M. D.; Mahler, B.; Lobo, R. P. S. M.; Dubertret, B.; Efros, A. L. Colloidal Nanoplatelets with TwoDimensional Electronic Structure. Nat. Mater. 2011, 10, 936-941.

10. Mahler, B.; Nadal, B.; Bouet, C.; Patriarche, G.; Dubertret, B. Core/shell Colloidal Semiconductor Nanoplatelets. J. Am. Chem. Soc. 2012, 134, 18591-18598.

11. Kunneman, L. T.; Tessier, M. D.; Heuclin, H.; Dubertret, B.; Aulin, Y. V.; Grozema, F. C.; Schins, J. M.; Siebbeles, L. D. A. Bimolecular Auger Recombination of Electron-Hole Pairs in Two-Dimensional CdSe and CdSe/CdZnS Core/Shell Nanoplatelets. J. Phys. Chem. Lett. 2013, 4, 3574-3578.

12. Tessier, M. D.; Spinicelli, P.; Dupont, D.; Patriarche, G.; Ithurria, S.; Dubertret, B. Efficient Exciton Concentrators Built from Colloidal Core/Crown CdSe/CdS Semiconductor Nanoplatelets. Nano Lett. 2014, 14, 207-213.

13. Biadala, L.; Liu, F.; Tessier, M. D.; Yakovlev, D. R.; Dubertret, B.; Bayer, M. Recombination Dynamics of Band Edge Excitons in Quasi-Two-Dimensional CdSe Nanoplatelets. Nano Lett. 2014, 14, 1134-1139.

14. Pelton, M.; Ithurria, S.; Schaller, R. D.; Dolzhnikov, D. S.; Talapin, D. V. Carrier Cooling in Colloidal Quantum Wells. Nano Lett. 2012, 12, 6158-6163.

15. Achtstein, A. W.; Schliwa, A.; Prudnikau, A.; Hardzei, M.; Artemyev, M. V.; Thomsen, C.; Woggon, U. Electronic Structure and Exciton-Phonon Interaction in Two-Dimensional Colloidal CdSe Nanosheets. Nano Lett. 2012, 12, 3151-3157.

16. Chen, Z.; Nadal, B.; Mahler, B.; Aubin, H.; Dubertret, B. Quasi-2D Colloidal Semiconductor Nanoplatelets for Narrow Electroluminescence. Adv. Funct. Mater. 2014, 24, 295-302.

17. She, C.; Fedin, I.; Dolzhnikov, D. S.; Demortière, A.; Schaller, R. D.; Pelton, M.; Talapin, D. V.; Richard, D. Low-Threshold Stimulated Emission Using Colloidal Quantum Wells. Nano Lett. 2014, 14, 2772-2777.

18. Guzelturk, B.; Kelestemur, Y.; Olutas, M.; Delikanli, S.; Demir, H. V. Amplified Spontaneous Emission and Lasing in Colloidal Nanoplatelets. ACS Nano 2014, 8, 6599-6605.

19. Abécassis, B.; Tessier, M. D.; Davidson, P.; Dubertret, B. SelfAssembly of CdSe Nanoplatelets into Giant MicrometerScale Needles Emitting Polarized Light. Nano Lett. 2014, 14, 710-715.

20. Tessier, M. D.; Biadala, L.; Bouet, C.; Ithurria, S.; Abecassis, B.; Dubertret, B. Phonon Line Emission Revealed by SelfAssembly of Colloidal Nanoplatelets. ACS Nano 2013, 7, 3332-3340.

21. Förster, T. Zwischenmolekulare Energiewanderung Und Fluoreszenz. Ann. Phys. 1948, 437, 55-75.

22. Kagan, C.; Murray, C.; Nirmal, M.; Bawendi, M. Electronic Energy Transfer in CdSe Quantum Dot Solids. Phys. Rev. Lett. 1996, 76, 1517-1520.

23. Crooker, S.; Hollingsworth, J.; Tretiak, S.; Klimov, V. Spectrally Resolved Dynamics of Energy Transfer in QuantumDot Assemblies: Towards Engineered Energy Flows in Artificial Materials. Phys. Rev. Lett. 2002, 89, 186802.
24. Guzelturk, B.; Martinez, P. L. H.; Zhang, Q.; Xiong, Q.; Sun, H.; Sun, X. W.; Govorov, A. O.; Demir, H. V. Excitonics of Semiconductor Quantum Dots and Wires for Lighting and Displays. Laser Photon. Rev. 2014, 8, 73-93.

25. Tessier, M. D. M. D.; Javaux, C.; Maksimovic, I.; Loriette, V.; Dubertret, B.; Kunneman, L. T.; Heuclin, H.; Aulin, Y. V.; Grozema, F. C.; Schins, J. M.; et al. Spectroscopy of Single CdSe Nanoplatelets. J. Phys. Chem. Lett. 2013, 4, 35743578.

26. Tessier, M. D.; Javaux, C.; Maksimovic, I.; Loriette, V.; Dubertret, B. Spectroscopy of Single CdSe Nanoplatelets. ACS Nano 2012, 6, 6751-6758.

27. Shirasaki, Y.; Supran, G. J.; Bawendi, M. G.; Bulović, V. Emergence of Colloidal Quantum-Dot Light-Emitting Technologies. Nat. Photonics 2012, 7, 13-23.

28. Anikeeva, P.; Madigan, C.; Halpert, J.; Bawendi, M.; Bulović, V. Electronic and Excitonic Processes in Light-Emitting Devices Based on Organic Materials and Colloidal Quantum Dots. Phys. Rev. B 2008, 78, 085434.

29. Yeltik, A.; Guzelturk, B.; Hernandez-Martinez, P. L.; Govorov, A. O.; Demir, H. V. Phonon-Assisted Exciton Transfer into Silicon Using Nanoemitters: The Role of Phonons and Temperature Effects in Förster Resonance Energy Transfer. ACS Nano 2013, 7, 10492-10501.

30. Pal, B. N.; Ghosh, Y.; Brovelli, S.; Laocharoensuk, R.; Klimov, V. I.; Hollingsworth, J. A.; Htoon, H. "Giant" CdSe/CdS Core/ shell Nanocrystal Quantum Dots as Efficient Electroluminescent Materials: Strong Influence of Shell Thickness on Light-Emitting Diode Performance. Nano Lett. 2012, 12, 331-336.

31. Kagan, C.; Murray, C.; Bawendi, M. Long-Range Resonance Transfer of Electronic Excitations in Close-Packed CdSe Quantum-Dot Solids. Phys. Rev. B 1996, 54, 86338643.

32. Akselrod, G. M.; Prins, F.; Poulikakos, L. V.; Lee, E. M. Y.; Weidman, M. C.; Mork, A. J.; Willard, A. P.; Bulović, V.; Tisdale, W. A. Subdiffusive Exciton Transport in Quantum Dot Solids. Nano Lett. 2014, 14, 3556-3562.

33. Novotny, L.; Hecht, B. Principles of Nano-Optics; Cambridge University Press, 2012.

34. Tessier, M. D.; Mahler, B.; Nadal, B.; Heuclin, H.; Pedetti, S.; Dubertret, B. Spectroscopy of Colloidal Semiconductor Core/Shell Nanoplatelets with High Quantum Yield. Nano Lett. 2013, 13, 3321-3328.

35. Thon, S. M.; Ip, A. H.; Voznyy, O.; Levina, L.; Kemp, K. W.; Carey, G. H.; Masala, S.; Sargent, E. H. Role of Bond Adaptability in the Passivation of Colloidal Quantum Dot Solids. ACS Nano 2013, 7, 7680-7688.

36. Hines, D. A.; Kamat, P. V. Quantum Dot Surface Chemistry: Ligand Effects and Electron Transfer Reactions. J. Phys. Chem. C 2013, 117, 14418-14426.

37. Guzelturk, B.; Hernandez Martinez, P. L.; Sharma, V. K.; Coskun, Y.; Ibrahimova, V.; Tuncel, D.; Govorov, A. O.; Sun, X. W.; Xiong, Q.; Demir, H. V.; et al. Study of Exciton Transfer in Dense Quantum Dot Nanocomposites. Nanoscale 2014, 6, 11387-11394.

38. Stoferle, T.; Scherf, U.; Mahrt, R. F. Energy Transfer in Hybrid Organic/Inorganic Nanocomposites. Nano Lett. 2009, 9, 453-456.

39. Greenham, N.; Peng, X.; Alivisatos, A. Charge Separation and Transport in Conjugated-Polymer/SemiconductorNanocrystal Composites Studied by Photoluminescence Quenching and Photoconductivity. Phys. Rev. B 1996, 54, 17628-17637.

40. Lutz, D. R.; Nelson, K. A.; Gochanour, C. R.; Fayer, M. D. Electronic Excited State Energy Transfer, Trapping by Dimers and Fluorescence Quenching in Concentrated Dye Solutions: Picosecond Transient Grating Experiments. Chem. Phys. 1981, 58, 325-334.

41. Kułak, L.; Bojarski, C. Forward and Reverse Electronic Energy Transport and Trapping in Solution. I. Theory. Chem. Phys. 1995, 191, 43-66.

42. Loring, R. F.; Andersen, H. C.; Fayer, M. D. Electronic Excited State Transport and Trapping in Solution. J. Chem. Phys. 1982, 76, 2015-2027. 
43. Lakowicz, J. R. Principles of Fluorescence Spectroscopy; Lakowicz, J. R., Ed.; Springer US: Boston, MA, 2006; Vol. 13, p 029901.

44. Menke, S. M.; Luhman, W. A.; Holmes, R. J. Tailored Exciton Diffusion in Organic Photovoltaic Cells for Enhanced Power Conversion Efficiency. Nat. Mater. 2013, 12, 152157.

45. Galland, C.; Ghosh, Y.; Steinbrück, A.; Sykora, M.; Hollingsworth, J. A.; Klimov, V. I; Htoon, H. Two Types of Luminescence Blinking Revealed by Spectroelectrochemistry of Single Quantum Dots. Nature 2011, 479, 203-207.

46. Rosen, S.; Schwartz, O.; Oron, D. Transient Fluorescence of the Off State in Blinking CdSe/CdS/ZnS Semiconductor Nanocrystals is Not Governed by Auger Recombination. Phys. Rev. Lett. 2010, 104, 157404.

47. Frantsuzov, P.; Kuno, M.; Jankó, B.; Marcus, R. A. Universal Emission Intermittency in Quantum Dots, Nanorods and Nanowires. Nat. Phys. 2008, 4, 519-522.

48. Brokmann, X.; Coolen, L.; Dahan, M.; Hermier, J. Measurement of the Radiative and Nonradiative Decay Rates of Single CdSe Nanocrystals through a Controlled Modification of Their Spontaneous Emission. Phys. Rev. Lett. 2004, 93, 107403.

49. Vietmeyer, F.; Tchelidze, T.; Tsou, V.i Janko, B.; Kuno, M. Electric Field-Induced Emission Enhancement and Modulation in Individual CdSe Nanowires. ACS Nano 2012, 6, 9133-9140.

50. Knowles, K. E.; McArthur, E. A.; Weiss, E. A. A Multi-Timescale Map of Radiative and Nonradiative Decay Pathways for Excitons in CdSe Quantum Dots. ACS Nano 2011, 5, 2026-2035.

51. Kunneman, L. T.; Schins, J. M.; Pedetti, S.; Heuclin, H.; Grozema, F. C.; Houtepen, A. J.; Dubertret, B.; Siebbeles, L. D. A. Nature and Decay Pathways of Photoexcited States in CdSe and CdSe/CdS Nanoplatelets. Nano Lett. 2014, 10.1021/nl503406a.

52. Powell, R. C.; Soos, Z. G. Singlet Exciton Energy Transfer in Organic Solids. J. Lumin. 1975, 11, 1-45. 